\title{
Evaluation of Malariometric indices of last 5 years in rural areas of Jamnagar district
}

\author{
Authors \\ Dr Aniruddha Gohel ${ }^{1}$, Dr Naresh Makwana ${ }^{2}$, Dr Mittal Rathod ${ }^{3}$, Dr Kishor Dhaduk ${ }^{4}$, \\ Dr Dipesh Parmar ${ }^{5}$ \\ ${ }^{1}$ Post Graduate, Community Medicine Dept., M.P.Shah GMC, Jamnagar \\ ${ }^{2}$ Professor, Community Medicine Dept., Shree M.P.Shah GMC, Jamnagar \\ ${ }^{3}$ Tutor, Community medicine Dept., M.P.Shah GMC, Jamnagar \\ ${ }^{4}$ Assistant Professor, Community Medicine Dept., Shree M.P.Shah GMC, Jamnagar \\ ${ }^{5}$ Professor \& Head, Community Medicine Dept., Shree M.P.Shah GMC, Jamnagar \\ Corresponding Author \\ Dr Aniruddha Gohel
}

Post Graduate, Community Medicine Dept., Shree M.P.Shah Medical College, Jamnagar, Gujarat, India.

Email: aniruddha.gohel198833@gmail.com, Mobile No.9924470547

\begin{abstract}
A long back i.e. in 1953 the NMCP was launched, with many major mile stones in the programme, reframed last in year 2003 as NVBDCP \& convergence under NRHM in 2005. Still we are facing the problems of morbidity as well as mortality from malaria. For reduction of so there must be well functioning health system as prescribed in NVBDCP. All defined targets must be fulfilled for better functioning of health care services and it would thus reduce malarial burden. A cross sectional study was conducted in selected PHC from each Taluka by simple random sampling. Data of the last five year shows that surveillance activity was improved over time. Mean API was reduced during last four years with highest mean API of 1.63 in the year 2010 to lowest (0.57) in the year 2013.Due to geographic location and migration of agricultural labourers, some PHCs were still strives to lower their API.

Key words: Malariometric Indices, PHC, Evaluation
\end{abstract}

\section{Introduction}

Malaria is still a major global public health concern, despite many countries especially in the endemic Afro-Asian settings, having paid a considerable focus on its control. ${ }^{(1)}$ India reports the highest malaria burden in the Southeast Asia region with $61 \%$ of the regional malaria cases. ${ }^{\text {(2) }}$

So long back i.e. in 1953 the NMCP was launched, with many major mile stones in the programme, reframed last in year 2003 as NVBDCP \& convergence under NRHM in 2005. Still we are facing the problems of morbidity as well as mortality from malaria. Data of the year 2012 clearly states that the goal of national health policy (3) as well as NVBDCP still not achieved. This may suggest, there may be a gap in ongoing programme in various components like technical constraints, operational constraints (inefficiency), managerial component \& community participation too. ${ }^{(4)}$ 
Targets of NVBDCP are Annual Blood Parasite Incidence (ABER) should be over $10 \%$, Annual Parasite Incidence (API) should be 1.3 or less. ${ }^{(5)}$ About $95 \%$ of the country's population resides in malaria endemic areas, and $80 \%$ of malaria reported in the country is confined to regions that have more than $20 \%$ of their population residing in tribal, hilly, difficult and inaccessible areas. The most affected states are: Andhra Pradesh, Chhattisgarh, Gujarat, Jharkhand, Karnataka, Madhya Pradesh, Maharashtra, Odisha, Rajasthan and West Bengal. ${ }^{(6)}$ Despite more than five decades of intensive control efforts, malaria is still a challenge to the Indian health system. ${ }^{(6)}$ The average cost of treatment was 475 rupees in 2012. Earnings loss per day was 213 rupees and the total work days lost is assumed to 10 days, derived from the NSS study. ${ }^{(7)}$ For reduction of so there must be well functioning health system as prescribed in NVBDCP. All defined targets must be fulfilled for better functioning of health care services and it would thus reduce malarial burden.

The study district is one of the endemic regions of the country. ${ }^{(8)}$ In the study district, in year 2012 total malaria cases were 1556 , of which 72 were P.falciparum cases. ${ }^{(9)}$ So in study district too, there may be various constraints. Keeping in view the above stated problem, there is a need to evaluate the Malariometric indices in study district.

\section{Objectives}

The primary objective of present study was to evaluate Malariometric indices in various primary health centres of study district.

\section{Materials and methods:}

Study area: The present study was conducted in rural areas of the study district.

Type of study: A cross-sectional study.

Study period: The study period was one year, i.e. From July 2013 to June 2014.

Study design: Out of the total talukas in study district, one PHC was selected from each taluka by simple random sampling technique.

Method of study: After selecting the PHCs, visits have been made to the PHCs. From each PHC, data regarding last five years Malariometric indices were collected in a pre-designed and pre-tested Proforma.

Ethical clearance: The study protocol was reviewed and approved by the institutional ethical committee of the institution. Prior consent was taken after fully explaining the purpose of the study.

Data entry and analysis: The data entry was done using Microsoft Office Excel 2007 and data analysis was done using EPI INFO and in Microsoft Office Excel 2007. Charts were prepared for showing trend of situation in District/PHC.

\section{Terms and Definitions Used In the Present Study Malariometric indices: ${ }^{(10)}$}

\section{Annual Blood smear Examination Rate (ABER)}

Number of blood smears examined in a year $* 100$

Total population

It is an index of operational efficiency of surveillance.

\section{Annual Parasite Index (API)}

Total No. of blood smears positive for malaria parasite in a year $\mathrm{x} 1000$

Total population

The most common index used at present is annual parasite incidence.

\section{Slide Positivity Rate (SPR)}

Total No. of blood smears found positive for malaria parasite $\mathrm{x} 100$

Total no. of blood smears examined

It is important, that SPR often bears a direct relation to ABER.

\section{P.falciparum percentage (Pf \%)}

Total no. of blood smears found positive for P.falciparum x 100

Total no. of blood smears positive for malaria parasite 
High risk area $=$ Where ABER is less than $10 \%$

API is more than 1.3

Poor working area $=$ Where ABER is less than $10 \%$ API is less than 1.3

Problematic zone $=$ Where ABER is more than $10 \%$ API is more than 1.3

Comfortable zone $=$ Where ABER is more than $10 \%$ API is less than 1.3

\section{Results And Discussion:}

Figure 1 shows, the malaria surveillance activities in selected ten PHCs were satisfactory (ABER>10\%) in the year 2012 and none of the PHCs belonged to high risk area or poor performing areas according to guideline (ABER $<10 \%$ and API $>1.3$ ). Padana and Nikava PHCs were identified as problematic and borderline problematic areas for Malaria because of their higher API. In the remaining PHCs endemicity of malaria was not a problem $(\mathrm{API} \leq 1.3$ and $\mathrm{ABER}$ $\geq 10$ ).

The data of ABER and API of two identified problematic PHCs suggests that the malaria disease is still endemic in their areas and malaria health care workers were collecting blood slides of fever cases during routine surveillance or by intensive fever survey and laboratory technicians could find positive malaria cases among them according to guideline. But in some PHCs having similar geographical and demographical situations (Surajkaradi, Samana and Gunda), the API was $<0.5$ which indicates either very low malarial endemicity or inauthentic blood slides collection and/or its examination by laboratory technicians.

Figure 2 shows, the malaria surveillance activities in selected ten PHCs were satisfactory (ABER>10\%) in the year 2013 and improved as compared to year 2012, except PHC Bhatia had annual blood examination rate just around $10 \%$. None of the PHCs belonged to high risk area or poor performing areas according to guideline (ABER $<10 \%$ and API $>1.3)$. Padana and Nikava PHCs were identified as problematic and borderline problematic areas for Malaria because of their higher API in the year 2012. But surveillance activity was improved. These PHCs were now in comfortable zone. In the remaining PHCs other than Padana and Nikava, endemicity of malaria was not a problem $(\mathrm{API} \leq 1.3$ and $\mathrm{ABER} \geq 10)$ in the year 2012 . But main point of concern is that one PHC Hadiana now in the borderline problematic zone as API just around 1.3.

The data of ABER and API of one identified problematic PHC suggests that the malaria disease is still endemic in their areas and malaria health care workers were collecting blood slides of fever cases during routine surveillance or by intensive fever survey and laboratory technicians could find positive malaria cases among them according to guideline. But in some PHCs having similar geographical and demographical situations (Surajkaradi, Samana, Aliabada, Bhatia, Nikava and Gunda), the API was $<0.5$ which indicates either very low malarial endemicity or inauthentic blood slides collection and/or its examination by laboratory technicians.

So, as compared to year 2012, the main findings giving rise to concern were that PHC Hadiana became borderline problematic area, surveillance activity was reduced in PHC Bhatia and PHC Vadatra was endemic for malaria (API just around lin the year 2012 and 2013).

Table 1 shows rate of annual blood examination in various PHCs from 2009 to 2013 period. Mean ABER ranges from 12.92 to 19.25 for the year 2009 to 2013.Data of the last five year shows that surveillance activity was improved over time.

Data of Gujarat state shows that ABER in the years 2011, 2012 and 2013 was 18.48, 17.48 and 20.23.ABER was improved over time in Gujarat state from 18.48 to 20.23 from 2011 to 2013.It was similar to findings of our study. ${ }^{(11)}$

Data of India shows that ABER in the years 2009, 2010, 2011 and 2012 was 8.99, 9.31, 9.12 and 9.ABER was same over time in from 2009 to 2011. It was below $10 \%$. $^{(11)}$

Table 2 shows annual parasite incidence in various PHCs from 2009 to 2013 period. Mean API was reduced during last four years with highest mean 
API of 1.63 in the year 2010 to lowest (0.57) in the year 2013.Due to geographic location and migration of agricultural labourers, some PHCs were still strives to lower their API.

Data of Gujarat state shows that API in the years 2011, 2012 and 2013 was $1.51,1.24$ and 0.93.API was improved over time in Gujarat state from 1.51 to 0.93 from 2011 to 2013.It was almost similar to findings of our study. ${ }^{(11)}$

Data of India shows that API in the years 2009, 2010, 2011 and 2012 was 1.36, 1.37, 1.10 and 0.88 . API was reduced over time in from 2009 to 2011. (11)

Above table shows that mean slide positivity rate was reducing from 1.08 in the year $2009-2010$ to 0.3 in the year 2013, which shows that the surveillance activity was quite good as mentioned in table 1.

Data of Gujarat state shows that SPR in the years 2011, 2012 and 2013 was $0.82 \%, 0.71 \%$ and $0.46 \%$. SPR was improved over time in Gujarat state from $0.82 \%$ to $0.46 \%$ from 2011 to 2013.It was almost similar to findings of our study. ${ }^{(11)}$
Data of India shows that SPR in the years 2009, 20102011 and 2012 was $1.51 \%, 1.47 \%, 1.10 \%$ and $0.98 \%$ SPR was reduced over time from 2009 to 2011. In contrast, present study shows SPR was much lower. ${ }^{(11)}$

Table 4 shows Pf\% in various PHCs from 2009 to 2013 period. With good mean slide positivity rate and mean blood examination rate number of malaria cases has reduced over time, but proportion of falciparum malaria cases has risen in the year 2013.This may be because importation of parasite from immigration of agricultural labourers.

Data of Gujarat state shows that Pf\% in the years 2011, 2012 and 2013 was 17.95\%, 13.75\% and $15.59 \%$. Pf\% was same over time in Gujarat state from 2011 to 2013. It was around 15\%. It was almost similar to findings of our study. ${ }^{(1)}$

Data of India shows that Pf\% in the years 2009, 2010, 2011 and 2012 was $53.72 \%, 52.15 \%, 50 \%$ and $50.01 \%$. Pf\% was same over time in from 2009 to 2011. Around half of the malaria cases were falciparum cases. In contrast, present study shows that Pf\% was much lower than national level. ${ }^{(11)}$

Figure 1: Performance of selected PHCs (2012)

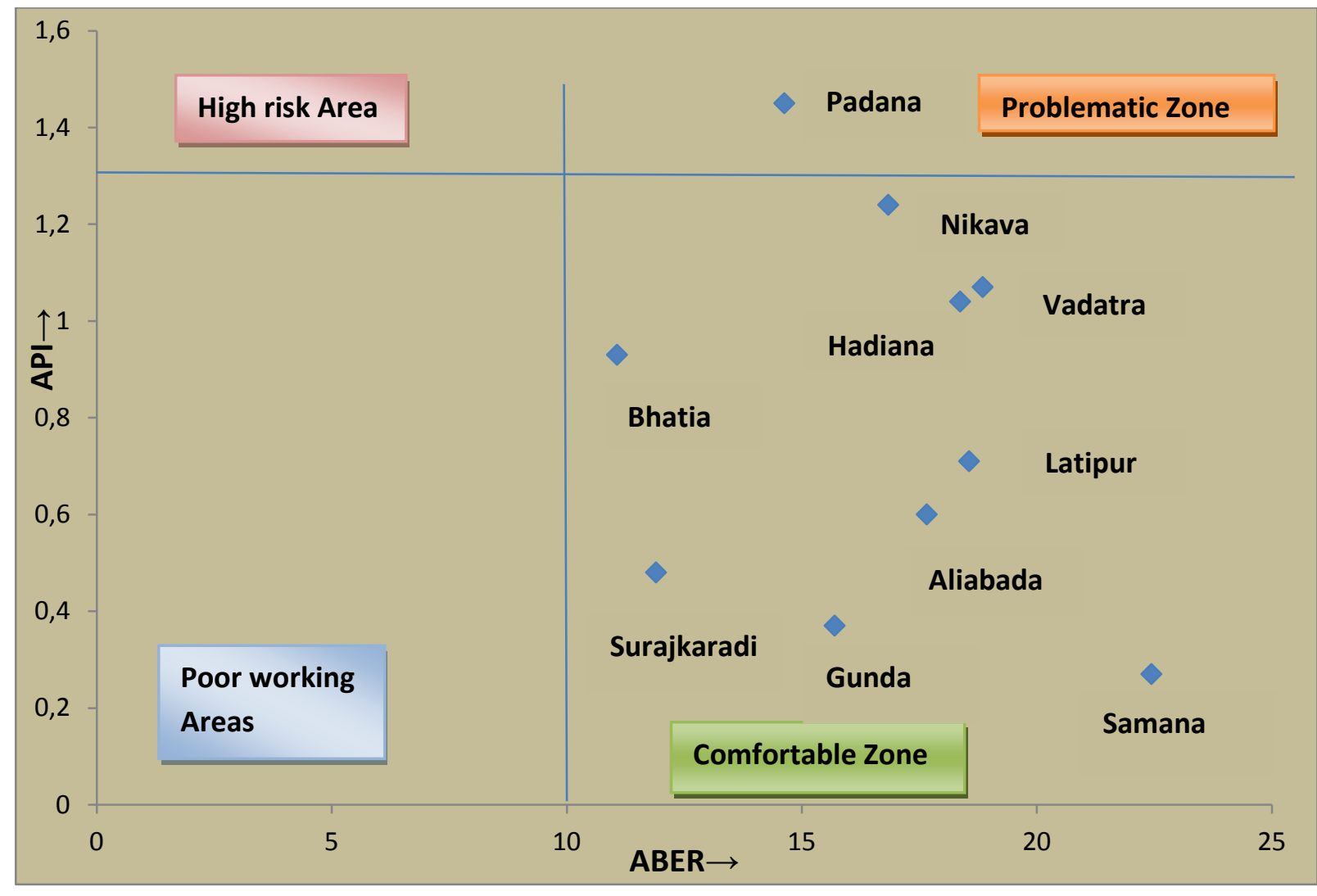




\section{JMSCR Vol.||03||Issue ||08||Page 7010-7016||August}

Figure 2: Performance of selected PHCs (2013)

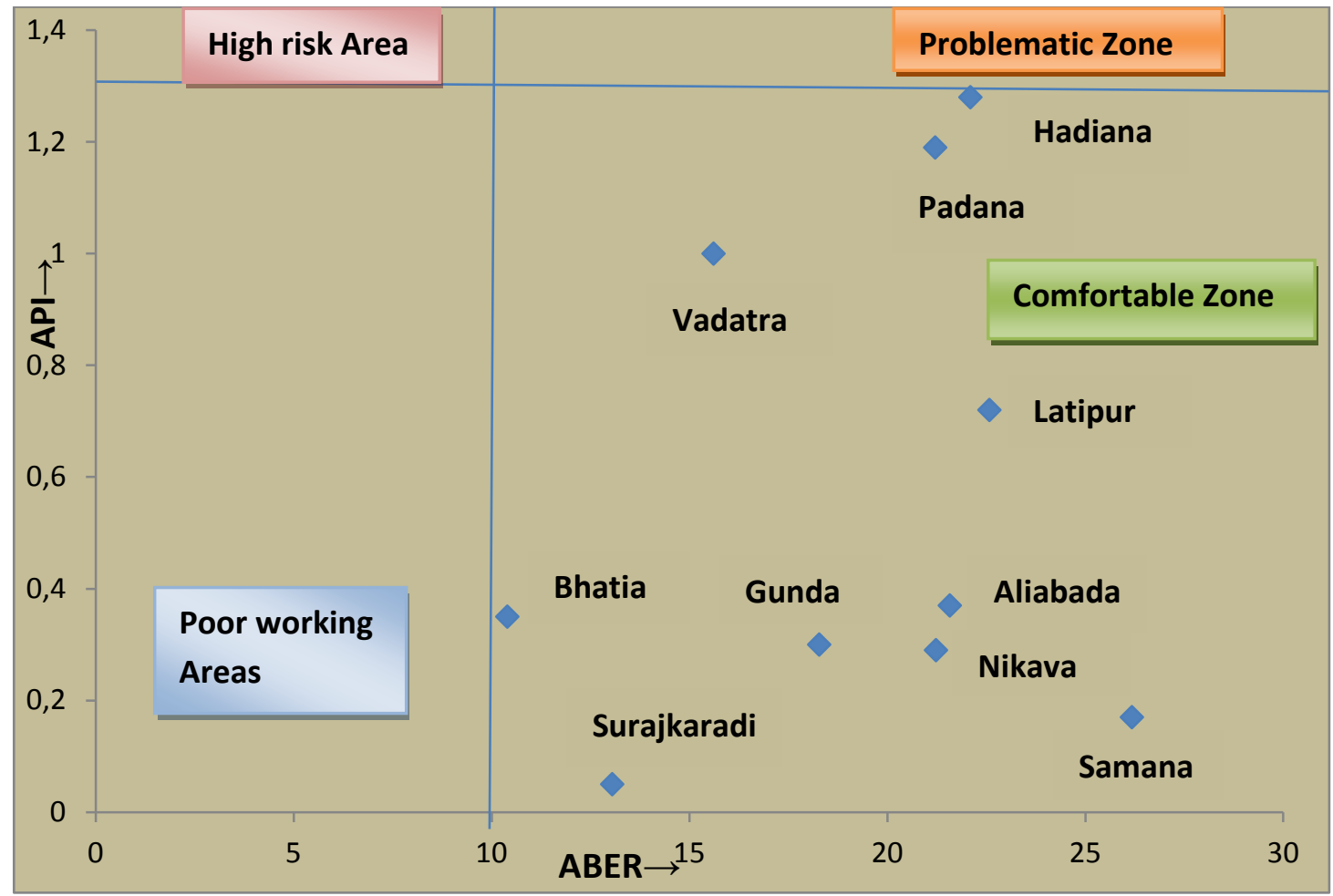

Table 1: ABER in the selected PHCs of study district

\begin{tabular}{|c|c|c|c|c|c|}
\hline PHC & 2009 & 2010 & 2011 & 2012 & 2013 \\
\hline Aliabada & 13.64 & 16.82 & 16.76 & 17.66 & 21.58 \\
\hline Bhatia & 8.24 & 9.69 & 10.85 & 11.07 & 10.40 \\
\hline Gunda & 12.60 & 11.50 & 13.93 & 15.70 & 18.28 \\
\hline Hadiana & 18.64 & 16.79 & 17.40 & 18.37 & 22.10 \\
\hline Latipur & 16.39 & 12.10 & 17.23 & 18.56 & 22.58 \\
\hline Nikava & 17.34 & 19.17 & 18.78 & 16.84 & 21.23 \\
\hline Padana & 8.62 & 13.21 & 13.12 & 14.63 & 21.21 \\
\hline Samana & 8.39 & 10.21 & 14.36 & 22.44 & 26.18 \\
\hline Surajkaradi & 5.72 & 10.26 & 11.97 & 11.90 & 13.05 \\
\hline Vadatra & 19.60 & 17.64 & 18.90 & 18.85 & 15.61 \\
\hline Mean & 12.13 & 13.86 & 15.34 & 16.45 & 19.22 \\
\hline SD & 4.97 & 3.53 & 2.86 & 3.41 & 4.86 \\
\hline
\end{tabular}


Table 2: API in the selected PHCs of study district

\begin{tabular}{|l|c|c|c|c|c|}
\hline PHC & $\mathbf{2 0 0 9}$ & $\mathbf{2 0 1 0}$ & $\mathbf{2 0 1 1}$ & $\mathbf{2 0 1 2}$ & $\mathbf{2 0 1 3}$ \\
\hline Aliabada & 1.42 & 1.39 & 1.55 & 0.60 & 0.37 \\
\hline Bhatia & 0.74 & 1.42 & 1.59 & 0.93 & 0.35 \\
\hline Gunda & 1.40 & 0.75 & 0.98 & 0.37 & 0.30 \\
\hline Hadiana & 2.16 & 1.66 & 1.64 & 1.04 & 1.28 \\
\hline Latipur & 0.81 & 0.9 & 1 & 0.71 & 0.72 \\
\hline Nikava & 2.27 & 3.26 & 2.56 & 1.24 & 0.29 \\
\hline Padana & 2.26 & 3.52 & 2.53 & 1.45 & 1.19 \\
\hline Samana & 0.26 & 0 & 0.31 & 0.27 & 0.17 \\
\hline Surajkaradi & 0.16 & 0.44 & 0.42 & 0.48 & 0.05 \\
\hline Vadatra & 1.85 & 2.98 & 2.36 & 1.07 & 1 \\
\hline Mean & 1.33 & 1.66 & 1.49 & 0.85 & 0.57 \\
\hline SD & 0.81 & 1.23 & 0.82 & 0.39 & 0.44 \\
\hline
\end{tabular}

Table 3: SPR in the selected PHCs of study district

\begin{tabular}{|l|c|c|c|c|c|}
\hline PHC & $\mathbf{2 0 0 9}$ & $\mathbf{2 0 1 0}$ & $\mathbf{2 0 1 1}$ & $\mathbf{2 0 1 2}$ & $\mathbf{2 0 1 3}$ \\
\hline Aliabada & 1.04 & 0.82 & 0.93 & 0.34 & 0.17 \\
\hline Bhatia & 0.9 & 1.47 & 1.46 & 0.84 & 0.34 \\
\hline Gunda & 1.12 & 0.65 & 0.70 & 0.23 & 0.16 \\
\hline Hadiana & 1.15 & 0.99 & 0.94 & 0.57 & 0.57 \\
\hline Latipur & 0.5 & 0.74 & 0.58 & 0.38 & 0.32 \\
\hline Nikava & 1.31 & 1.7 & 1.35 & 0.74 & 0.14 \\
\hline Padana & 2.61 & 2.66 & 1.93 & 0.99 & 0.56 \\
\hline Samana & 0.3 & 0 & 0.21 & 0.12 & 0.07 \\
\hline Surajkaradi & 0.28 & 0.43 & 0.34 & 0.40 & 0.04 \\
\hline Vadatra & 1.60 & 1.69 & 1.25 & 0.58 & 0.63 \\
\hline Mean & 1.082 & 1.13 & 0.97 & 0.53 & 0.3 \\
\hline SD & 0.69 & 0.79 & 0.53 & 0.28 & 0.22 \\
\hline
\end{tabular}

Table 4: $\mathrm{PF} \%$ in the selected PHCs of study district

\begin{tabular}{|l|c|c|c|c|c|}
\hline PHC & $\mathbf{2 0 0 9}$ & $\mathbf{2 0 1 0}$ & $\mathbf{2 0 1 1}$ & $\mathbf{2 0 1 2}$ & $\mathbf{2 0 1 3}$ \\
\hline Aliabada & 1.78 & 1.54 & 0 & 0 & 0 \\
\hline Bhatia & 2.04 & 2.94 & 0 & 0 & 0 \\
\hline Gunda & 0 & 4.16 & 0 & 0 & 22.22 \\
\hline Hadiana & 9.21 & 1.72 & 3.5 & 0 & 0 \\
\hline Latipur & 17.77 & 11.76 & 1.72 & 7.31 & 2.77 \\
\hline Nikava & 26.96 & 13.74 & 18.44 & 6 & 0 \\
\hline Padana & 9.19 & 9.80 & 2.72 & 1.59 & 20.41 \\
\hline Samana & 10 & 0 & 0 & 0 & 33.33 \\
\hline Surajkaradi & 12.5 & 0 & 0 & 4.16 & 0 \\
\hline Vadatra & 0.81 & 1.86 & 1.72 & 2.54 & 2.54 \\
\hline Mean & 9.0 & 4.7 & 2.81 & 1.6 & 8.1 \\
\hline SD & 8.57 & 5.07 & 5.64 & 2.77 & 12.35 \\
\hline
\end{tabular}




\section{Conclusion}

Data of the last five year shows that surveillance activity was improved over time.

Mean API was reduced during last four years with highest mean API of 1.63 in the year 2010 to lowest (0.57) in the year 2013.Due to geographic location and migration of agricultural labourers, some PHCs were still strives to lower their API.

With good mean slide positivity rate and mean blood examination rate number of malaria cases has reduced over time, but proportion of falciparum malaria cases has risen in the year 2013.This may be because importation of parasite from immigration of agricultural labourers.

\section{References}

1. World malaria report. Geneva : World Health Organization, 2008.

2. World malaria report. Geneva : World Health Organization, 2012.

3. J.Kishore. National Health Programs of India, 10th edition, page no.627 -.

4. Project implementation plan, National vector borne disease control programme support project under world bank on malaria control and kala azar.

5. J. koshore, National health programs of India, Textbook of preventive and social medicine, 10th edition page no.328.

6. National vector borne disease control programme,Ministry of Health and Family Welfare,GOI. [Online] http://nvbdcp.gov.in/malaria3.html. [Online]

7. Economic burden of malaria in India: The need for effective spending.Indrani Gupta, Samik Chowdhury. s.l. : WHO South-East Asia Journal of Public Health | JanuaryMarch 2014 | 3 (1).

8. Trends of malaria situation in jamanagar district, http://www.sco-pemed.org/f ulltextpdf.php?mno=43563.

9. National Vector Borne Disease Control Programme, Ministry of Health and Family Welfare, GOI. [ONLINE] http://www.nvbdcp.gov.in/malaria2.html .
10. Training module for medical officers, Primary health centre, NVBDCP, MOHFW,GOI.

11. National Vector Borne Disease Control Programme (NVBDCP), Ministry of Health and Family Welfare, GOI. [Online] www.nvbdcp.com.

\section{Abbreviations}

NMCP, NRHM, NVBDCP, NSS, ABER, API, SPR, PHC, PF\%

NMCP-National Malaria Control Programme

NRHM-National Rural Health Mission

NVBDCP- National vector borne disease control programme

NSS-National Sample Survey

ABER-Annual Blood Examination Rate

API-Annual Parasite Incidence

SPR-Slide Positivity Rate

PHC-Primary Health Centre

PF\%-P.Falciparum Percentage 